\title{
A Tale of Two Parasites: statistical modelling to support disease control programmes in Africa
}

\author{
Peter J Diggle \\ (Lancaster University and Health Data Research UK) \\ Emanuele Giorgi \\ (Lancaster University), \\ Julienne Atsame (Ministry of Health, Gabon), \\ Sylvie Ntsame Ella (Ministry of Health, Gabon), \\ Kisito Ogoussan (Task Force for Global Health) \\ and \\ Katherine Gass \\ (Task Force for Global Health)
}

August 26, 2019

\begin{abstract}
Vector-borne diseases have long presented major challenges to the health of rural communities in the wet tropical regions of the world, but especially in sub-Saharan Africa. In this paper we describe the contribution that statistical modelling has made to the global elimination programme for one vector-borne disease, onchocerciasis.

We explain why information on the spatial distribution of a second vector-borne disease, Loa loa, is needed before communities at high risk of onchocerciasis can be treated safely with mass distribution of ivermectin, an antifiarial medication.

We show how a model-based geostatistical analysis of Loa loa prevalence survey data can be used to map the predictive probability that each location in the region of interest meets a WHO policy guideline for safe mass distribution of ivermectin and describe two applications: one is to data from Cameroon that assesses prevalence using traditional blood-smear microscopy; the other is to Africa-wide data that uses a low-cost questionnaire-based method.

We describe how a recent technological development in image-based microscopy has resulted in a change of emphasis from prevalence alone to the bivariate spatial distribution of prevalence and the intensity of infection amongst infected individuals. We discuss how statistical
\end{abstract}


modelling of the kind described here can contribute to health policy guidelines and decisionmaking in two ways. One is to ensure that, in a resource-limited setting, prevalence surveys are designed, and the resulting data analysed, as efficiently as possible. The other is to provide an honest quantifi

cation of the uncertainty attached to any binary decision by reporting predictive probabilities that a policy-de

fined condition for action is or is not met.

Vector-borne diseases have long presented major challenges to the health of rural communities in the wet tropical regions of the world, but especially in sub-Saharan Africa. In this paper we describe the contribution that statistical modelling has made to the global elimination programme for one vector-borne disease, onchocerciasis.

We explain why information on the spatial distribution of a second vector-borne disease, Loa loa, is needed before communities at high risk of onchocerciasis can be treated safely with mass distribuiton of ivermectin, an antiflarial medication.

We show how a model-based geostatistical analysis of Loa loa prevalence survey data can be used to map the predictive probability that each location in the region of interest meets a WHO policy guideline for safe mass distribution of ivermectin and describe two applications: one to data from Cameroon that assesses prevalence using traditional blood-smear microscopy; one to Africa-wide data that uses a low-cost questionnaire-based method.

We describe how a recent technological development in image-based microscopy has resulted in a change of emphasis from prevalence alone to the bivariate spatial distribution of prevalence and the intensity of infection amongst infected individuals.

We discuss how statistical modelling of the kind described here can contribute to health policy guidelines and decision-making in two ways. One is to ensure that, in a resourcelimited setting, prevalece surveys are designed, and the resulting data analysed, as efficiently as possible. The other is to provide an honest quantification of the uncertainy attached to any binary decision by reporting predictive probabilities that a policy-defined condition for action is or is not met.

\section{Problem statement}

Vector-borne diseases have long presented major challenges to the health of rural communities in the wet tropical regions of the world, but especially in sub-Saharan Africa. In this paper we describe the contribution that statistical modelling has made to the global elimination programme for one vector-borne disease, onchocerciasis.

Onchocerciasis was not always targeted for elimination. When the International Task Force for Disease Eradication met in 1993, onchocerciasis was not among the six diseases considered eradicable at the time (Centers for Disease Control and Prevention, 1993). Indeed, the Onchocerciasis Control Program (OCP), and the African Programme for Onchocerciasis Control 
(APOC) that succeeded it, focused on controlling significant disease of the skin and eyes so that onchocerciasis would no longer be a public health problem (Amazigo, 2008). That strategy for control relied on mass drug administration (MDA) with ivermectin, an antifilarial medication that kills larval-stage parasites (microfilariae) before they can either cause significant disease in infected people or be transmitted to the blackfly vectors of infection. Ivermectin, under its trade name MECTIZAN ${ }^{\mathrm{R}}$, has been provided free of charge since 1987 by its manufacturer, Merck \& Co Inc, to control onchocerciasis in all affected countries world-wide ${ }^{1}$. Even so, the successful execution of a control programme using mass drug administration with ivermectin (henceforth, MDA) faced formidable practical difficulties due to the size and inaccessibility of the populations at risk and the low national income levels of many of the affected countries. To address this challenge and ensure that even the most remote populations can be reached, APOC developed a novel delivery strategy known as Community Directed Treatment with Ivermectin (CDTI, Homeida et al, 2002), which has since been used by onchocerciasis programmes with great success.

The past two decades have seen tremendous success for onchocercaisis programmes. In the Americas, where the infection is more focal and political will is strong, onchocerciasis has now been eliminated in 11 of 13 foci of infection over 6 countries (World Health Organisation, 2013, 2014, 2015; Thiele et al, 2016). In Africa, over a dozen annual rounds of MDA led to the interruption of transmission in selected foci in several countries including Mali, Senegal, Uganda, Ethiopia, Nigeria and Sudan (World Health Organisation, 2018). Collectively, these experiences demonstrated that the tools to detect and eliminate onchocerciasis infection exist and can be used successfully by country programmes. As a consequence, in 2012 the World Health Organization recognized that elimination is the appropriate target for onchocerciasis in Africa, where $99 \%$ of the world's population at risk live. This elimination effort is now being coordinated by WHO's regional office in Africa through their Expanded Special Project for Elimination of Neglected Tropical Diseases (ESPEN, World Health Organisation, 2012).

In the midst of this great progress, there remains one insidious obstacle to the global elimination of onchocerciasis, namely the presence of Loa loa. Towards the end of the last century, reports emerged of severe, sometimes fatal, reactions to ivermectin experienced by some individuals who were heavily infected with a second vector-borne parasitic infection, Loa loa (Gardon et al, 1997; Boussinesq et al, 1998; Boussinesq et al, 2003). This discovery threatened to de-rail the MDA progamme that until then had been regarded as safe. Because Loa loa was not considered to be a major public health problem in its own right, there was limited understanding of its geographical distribution in areas endemic for onchocerciasis, or of the numbers of highly infected individuals considered to be at risk of experiencing serious adverse reactions (henceforth, SAEs) to ivermectin. The presence of Loa loa has stymied the elimination effort in areas where the two parasites are co-endemic, as there has not been an agreed upon strategy for how to deliver safe treatment with ivermectin. The remainder of the paper describes how statistical modelling has been, and continues to be, used to address the "Loa loa problem," and hence to inform the operational policy around MDA for the elimination of onchocerciasis.

\footnotetext{
${ }^{1}$ See: https://investors.merck.com/news/press-release-details/2017/Merck-Commemorates-30 -Years-of-MECTIZAN- Donation- Program-Progress/default.aspx
} 


\section{Mapping Loaloa parasitological prevalence}

To make safe ivermectin treatment decisions for onchocerciasis in areas where Loa loa is potentially co-endemic, it is first necessary to map the areas where heavily infected individuals, that is individuals harboring an abundant number of Loa loa parasites, are likely to be found. A general feature of the epidemiology of vector-borne diseases is that heavily infected individuals are more likely to be found in high-prevalence areas. For evidence in the current context, see Boussinesq et al (2001). For this reason, and because it would have been infeasible to test individuals for their levels of infection with Loa loa parasites before administering iveremctin, Thomson et al (2000) used data from Loa loa prevalence surveys in conjunction with satellite-derived images of environmental variables to map the geographical variation in Loa loa prevalence. They fitted a logistic regression model to data on the presence/absence of Loa loa parasites under microscopic examination of blood smears for " 14,305 individuals sampled from more than 100 villages" in five Loa loa-endemic west and central African countries. As explanatory variables, they used data at $1 \mathrm{~km}$ pixel resolution on forest cover, land use type and soil type. Their model explained approximately $50 \%$ of the variation in prevalence over the sampled villages. Thomson et al (2004) also developed a logistic regression model for Loa loa prevalence using data for 14,225 individuals from 94 villages in Cameroon together with a wider range of explanatory variables; their model explained approximately $60 \%$ of the variation in prevalence over the sampled villages.

Variation in prevalence of an environmentally driven disease typically obeys Tobler's so-called first law of geography, which states that "everything is related to everything else, but near things are more related than distant things" (Tobler, 1970). The statistical expression of this is that empirical prevalence data usually exhibit spatial correlation. To the extent that the relevant environmental risk-factors can be measured, this spatial correlation can be removed by covariate adjustment, but this typically leaves an unexplained component of geographical variation that manifests itself in residual spatial correlation. One way to account for this residual spatial correlation is to extend the logistic regression model by including a latent, spatially correlated process in the linear predictor (Breslow and Clayton, 1993; Diggle, Moyeed and Tawn, 1998). The resulting model for $P(x)$, the prevalence of disease at location $x$, is

$$
\log [P(x) /\{1-P(x)\}]=d(x)^{\prime} \beta+S(x)
$$

where $d(x)$ is a vector of covariates associated with $x$ and $S(x)$ is a latent, spatially continuous stochastic process. Conditional on the prevalence surface $P(x)$ throughout the region of interest, the numbers $Y_{i}$ of infected individuals out of $m_{i}$ tested at locations $x_{i}: i=1, \ldots, n$ are independent, binomially distributed random variables with binomial probabilities $P\left(x_{i}\right)$ and denominators $m_{i}$.

Diggle, Thomson et al (2007) used this approach to refine the predictions in Thomson et al (2004), using prevalence data for 21,938 individuals from 168 villages in Cameroon and Nigeria. The addition of the stochastic term $S(x)$ on the right hand side of (??) helps to explain the geographical variation in Loa loa prevalence. Its purpose is two-fold: to give more robust inferences about the effects of the environmental risk-factors and, more importantly in this application, to give predictions of prevalence at unsampled locations that are both more 

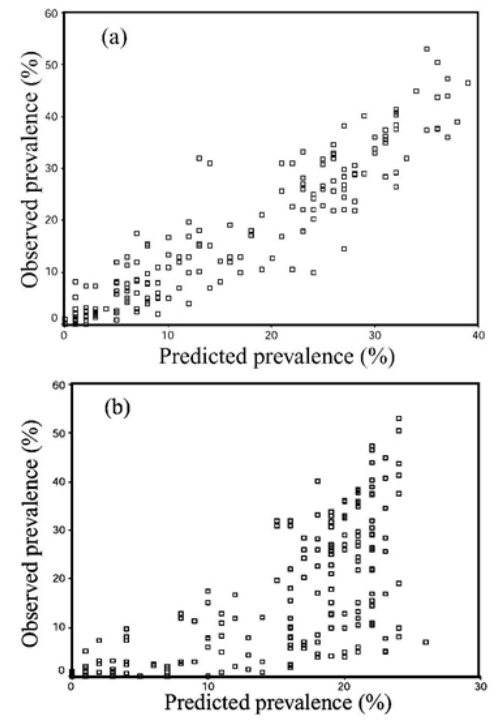

FIG. 3. The observed prevalences of Loa loa microfilaraemia plotted ag ans

Figure 1: Observed and predicted prevalences of Loa loa: (a) spatial model from Diggle, Thomson et al (2007); (b) logistic regression model from Thomson et al (2004). Figure reproduced from Diggle, Thomson et al, (2007).

accurate than can be obtained using only the available environmental risk-factors and more honest in their associated measures of uncertainty. Diggle, Thomson et al (2007) examined the residuals from a standard logistic regression model to suggest a suitable model for $S(x)$. This led them to specify $S(x)$ to be a Gaussian process with covariance function

$$
\operatorname{Cov}\left\{S(x), S\left(x^{\prime}\right)\right\}=\sigma^{2} \exp \left(-\left\|x-x^{\prime}\right\| / \phi\right)+\tau^{2} I\left(x=x^{\prime}\right),
$$

where $\|\cdot\|$ denotes distance and $I(\cdot)$ is the indicator function. This model recognises two sources of extra-binomial variation in the data, a spatial component $\sigma^{2}$ and a non-spatial component $\tau^{2}$. Diggle, Thomson et al (2007) fitted the model using an MCMC implementation of Bayesian inference, with the ratio $\tau^{2} / \sigma^{2}$ held fixed at 0.4 (owing to a limitation of the software available to the authors at the time), an improper uniform prior for $\sigma^{2}$ and the regression parameters $\beta$, and a proper uniform prior for the spatial correlation range parameter $\phi \sim \mathrm{U}(0, c)$ with $c=100 \mathrm{~km}$. The explanatory variables in the model were elevation and two satellite-derived variables associated with greenness of vegetation. The rationale for choosing these was that the vector, a Chrysops fly species, can breed successfully in hot, wet conditions; in equatorial regions, elevation acts as a proxy for minimum temperature.

The minimum mean square error predictor for $P(x)$ is its conditional expectation given the data. The improvement in accuracy of prediction over the logistic regression model of Thomson et al (2004) is summarised by the two scatterplots of observed against predicted prevalence shown in Figure ??. A conventional measure of the associated uncertainty would be the conditional variance of $P(x)$ given the data. Diggle, Thomson et al (2007) argue that a more useful way to summarise uncertainty is to map selected points of the predictive distribution of each $P(x)$, i.e. its conditional distribution given the data. This was particularly relevant 


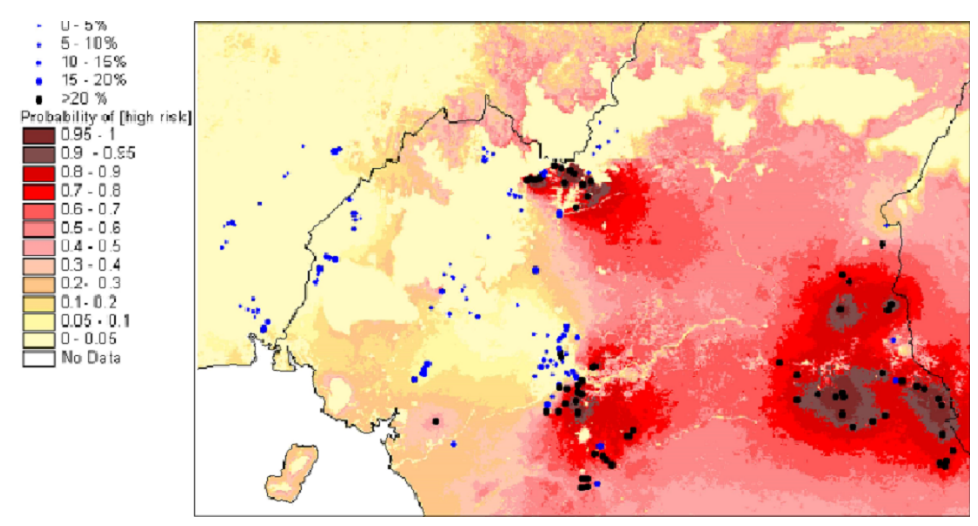

Figure 2: Map of the predictive probability that prevalence of Loa loa exceeds 0.2 (20\%). Solid dots indicate the sampled village locations and their observed prevalences. Figure reproduced from Diggle, Thomson et al, 2007.

to the application because by this time APOC policies included a requirement that in areas where Loa loa prevalence exceeded $0.2(20 \%)$, precautionary measures should be put in place before implementation of MDA with evermectin, so as to be able to respond promptly to any cases of SAEs. Accordingly, Figure ?? maps the predictive probability that $P(x)>0.2$. One striking feature of this map is that even in some areas where no data on prevalence are available, the model can be almost certain that $P(x)$ does not exceed 0.2 , whereas it can only be confident that $P(x)$ does exceed 0.2 in areas where prevalences above 0.2 have been observed. Put another way, the environmental variables alone can be sufficient to declare an area "safe" for MDA with ivermectin, but not to declare an area "unsafe." Finally, and importantly, large swathes of the map in Figure ?? are pink, representing a close to fifty-fifty call on prevalence exceeding 0.2. This is both the strength and the weakness of the map. Its strength is it honesty in declaring an answer of "don't know" to the policy-relevant question. Its weakness is the extent of the pink regions, indicating a need for substantially more data.

\section{Scaling up: the RAPLOA method}

The conclusion from the Diggle, Thomson et al (2007) analysis is that the intensity of datacollection in Cameroon is insufficient to delineate the whole of the country into "safe" and "unsafe" areas for MDA. But expanding even this intensity of data collection to all of the APOC countries would have been unaffordable. There was therefore an urgent need to develop a low-cost alternative to blood-smear microscopy for estimating village-level prevalence. Such an alternative had been proposed by Takougang et al (2002), and was in the process of being validated at the time of the Diggle, Thomson et al (2007) study. This method, RAPLOA, consisted of a three-item questionnaire: have you had past experience of eye worm? did it look like this photograph (of an adult worm in the white part of the eye)? did the most recent episode last between 1 and 7 days? Declaring a positive RAPLOA result as three "yes" answers gives village-level prevalence estimates that correlate well with estimates based on blood smear microscopy, at a fraction of the cost. A validation study appeared in 2012 (Wanji 

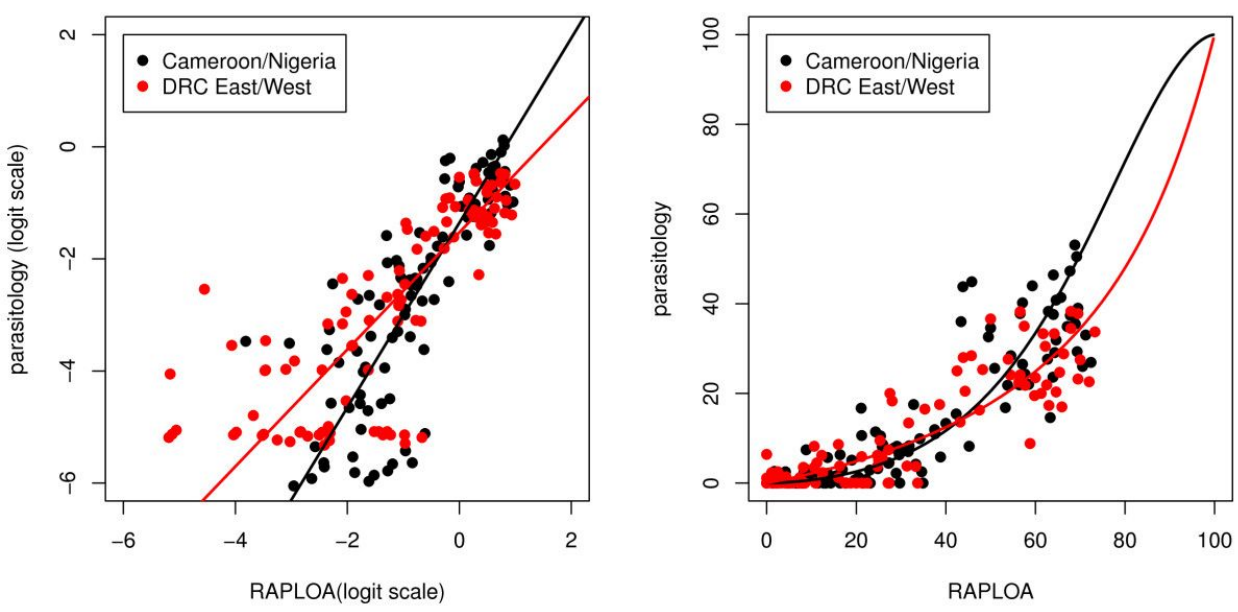

Figure 3: Relationship between village-level blood smear prevalence and RAPLOA prevalence. Black and red lines show the calibration models fitted to the original and validation data, respectively. The left-hand panel shows the relationship on the log-odds scale, the right-hand panel on the prevalence scale. Figure reproduced from Wanji et al (2012). Original data from Takougang et al (2002)

et al, 2012) although the data underlying that study had been collected and the results of a preliminary analysis reported to APOC some years previously, leading to the adoption of a policy guideline equating 40\% RAPLOA prevalence to $20 \%$ blood smear prevalence. In fact, the results in Wanji et al (2012) suggested a best-guess equivalence slightly higher than $40 \%$ but by this time the 40-20 rule had become well-established and changing this to one that implied a less stringent safety threshold would not have been acceptable. The discrepancy arose because the original analysis assumed a quadratic relationship between RAPLOA and bood smear prevalences, whereas Wanji et al (2012) assumed a linear relationship between log-odds-transformed prevalences; see Figure ??.

Zoure et al (2011) report on an Africa-wide study of RAPLOA prevalence, covering 381,575 individuals in 4,798 villages over an area of approximately 2500 by $3000 \mathrm{~km}$ in sub-Saharan Africa. The two panels of Figure ?? map the locations of the 4,798 villages and the predictive probability that RAPLOA prevalence exceeds $40 \%$

\section{Joint analysis of prevalence and intensity of infection: the LoaScope}

A major limitation of prevalence mapping as a way of identifying areas that are and are not considered to be safe for MDA with ivermectin is that village-level prevalence is at best a proxy for the proportion of village inhabitants who are heavily infected with Loa loa, and therefore at risk of experiencing an SAE when given ivermectin. Schlüter et al (2016) addressed this by investigating in more detail than hitherto the joint distribution of village-level prevalence and the distribution of levels of infection. They used data from Takougang et al (2002) and Wanji 

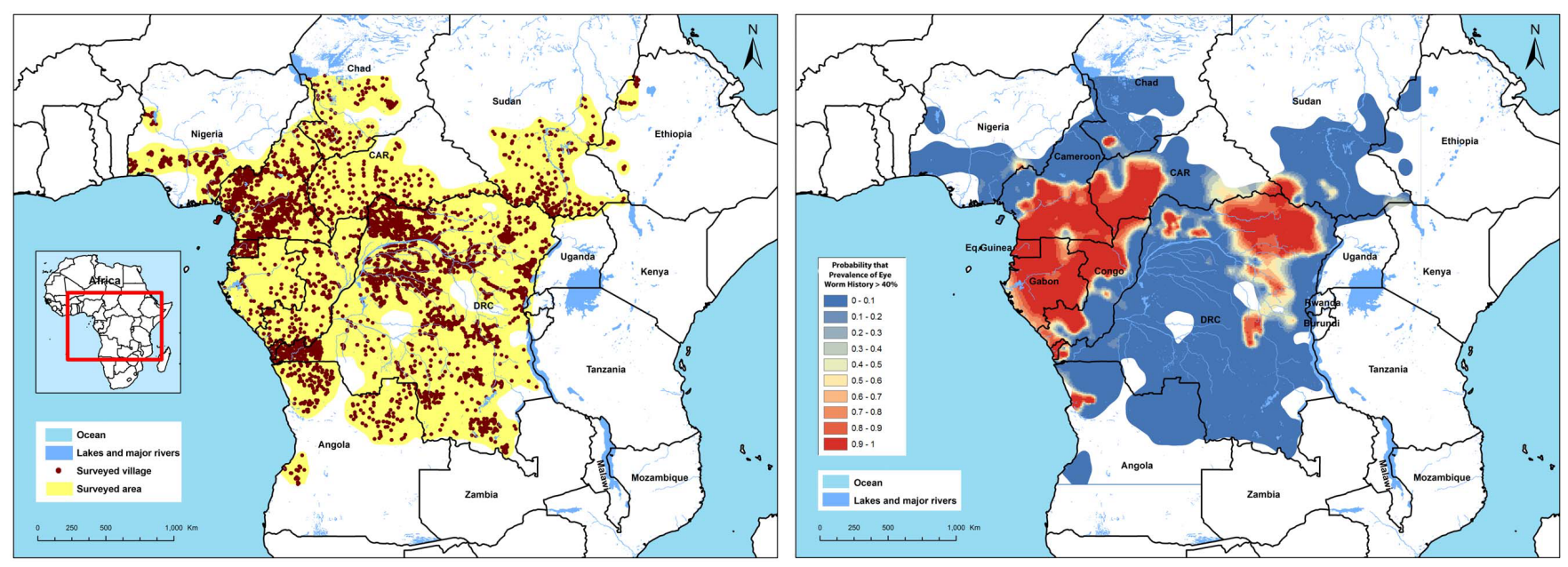

Figure 4: Maps of survey locations (left-hand panel) and the predictive probabiity that RAPLOA prevalence exceeds $40 \%$ (right-hand panel). Figure reproduced from Zoure et al (2011).

et al (2012) consisting of blood smear counts of Loa loa microfilariae per ml obtained from 19,049 individuals in 222 villages in Cameroon, Republic of Congo and Democratic Republic of Congo. They found that in most of the sampled villages the empirical distribution of microfilariae per ml was reverse-J-shaped, and that in villages with higher observed prevalence the distribution of positive counts typically had a longer upper tail; see Figure ??.

The behaviour illustrated in Figure ?? led Schlüter et al (2016) to propose a bivariate random effects model of the following form. Let $Y$ denote the number of microfilariae per $\mathrm{ml}$ for a randomly sampled individual in a particular village, and $(U, V)$ a latent bivariate random variable. Conditional on $(U, V)$, the distribution function of $Y$ is

$$
F(y)=1-P \times \exp \left\{-(y / \Lambda)^{\kappa}\right\}: y \geq 0
$$

where $\log \{P /(1-P)\}=U, \log \Lambda=V$ and the latent variable $(U, V)$ follows a bivariate Normal distribution, realised independently in different villages. Schlüter et al (2016) found, unsurprisingly, that in the fitted model $U$ and $V$ were positively correlated ( $95 \%$ confidence interval 0.534 to 0.864 ). This implies that it is possible to predict the proportion of highly infected, and therefore high-risk, individuals in a village, given only the presence or absence of Loa loa microfilariae in blood smears from a sample of village inhabitants. In most villages, the infection levels considered to be indicative of a high-risk individual lie well into the upper tail of the distribution. For this reason, model-based predictions based only on the sample size and number of presences of Loa loa microfilariae were more precise than empirical predictions based on the observed proportion of highly infected individuals.

Model-based predictions are, however, less robust than empirical ones, leading to an understandable reluctance to adopt them for routine use. A recent and potentially game-changing technological development has been the invention of the LoaScope, a form of portable microscopy that uses image analysis of the movement of microfilariae in an individual's blood sample to estimate their level of infection (D'Ambrosio et al, 2015). The practical significance 


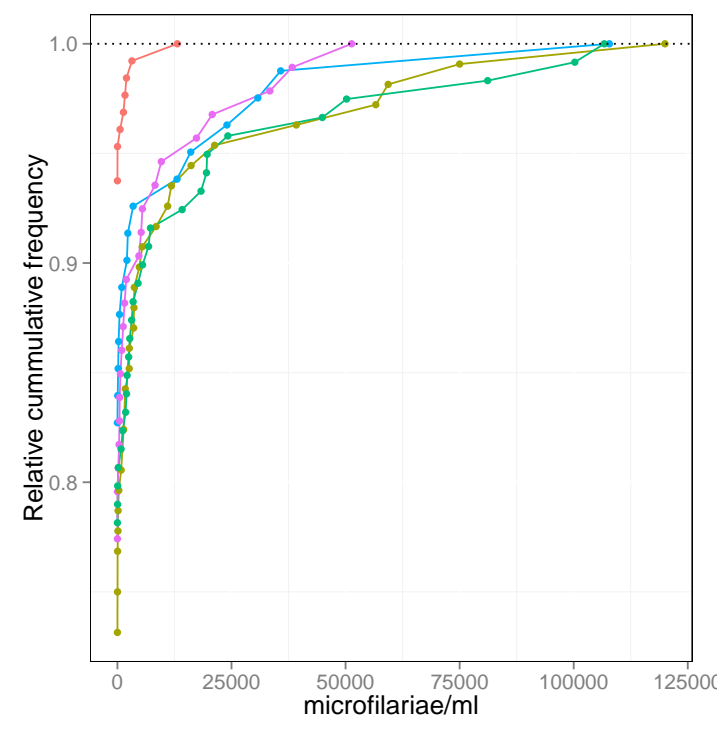

Figure 5: Empirical cumulative distributions of Loa loa microfilariae per $\mathrm{ml}$ in five randomly selected villages. Figure reproduced from Schlüter et al (2016).

of this is that it enables individual-level testing to identify high-risk individuals at the point of care and in real-time, without the need for trained microscopists. Kamgno et al (2017) propose using the LoaScope in a test-and-not-treat strategy whereby individuals are tested and ivermectin with-held if their estimated level of infection exceeds an agreed safe limit, currently set at 20,000 microfilariae per ml. While this test-and-not-treat strategy is presently the best tool for making safe ivermectin treatment decisions, the need to test every individual makes it a resource-intensive approach. The Schlüter et al (2016) model can be applied at village-level using LoaScope data from a sample of individuals to predict, with quantifiable uncertainty, whether the village is safe to treat with ivermectin. The potential value of this is that it can reduce the time and effort needed to establish whether MDA can be used in a particular village.

The statistical argument is as follows. Suppose it would be acceptable to declare a village safe for MDA if at most $k$ individuals (where $k$ may be zero or a small positive integer) would give a LoaScope response in excess of 20,000 microfilariae per ml. Call this event $T$. The Schlüter et al (2016) model can be used to calculate the predictive probability of $T$ given a set of LoaScope estimates $y=\left(y_{1}, \ldots, y_{n}\right)$. If this probability is at least $q$, for an agreed value of $q$ close to 1 , declare the village safe. If it is less than a second agreed value $q^{\prime}$ close to zero, declare it unsafe. If $q^{\prime}<\mathrm{P}(T \mid y)<q$, increase the sample size. Provided that the village population size, $N$ say, is known, then with $k=0$ this strategy converges to test-and-not-treat as $q$ approaches 1 . If $N$ is unknown, the model can only calculate the probability than an unsampled individual will give a LoaScope estimate in excess of 20,000 microfilariae per ml.

Giorgi et al (2017) extended the Schlüter et al (2016) model by allowing the random effects $(U, V)$ to be spatially correlated. Incorporating an estimate of the correlation structure of a bivariate spatial process, $\{U(x), V(x)\}$ leads to more precise prediction at any one location 

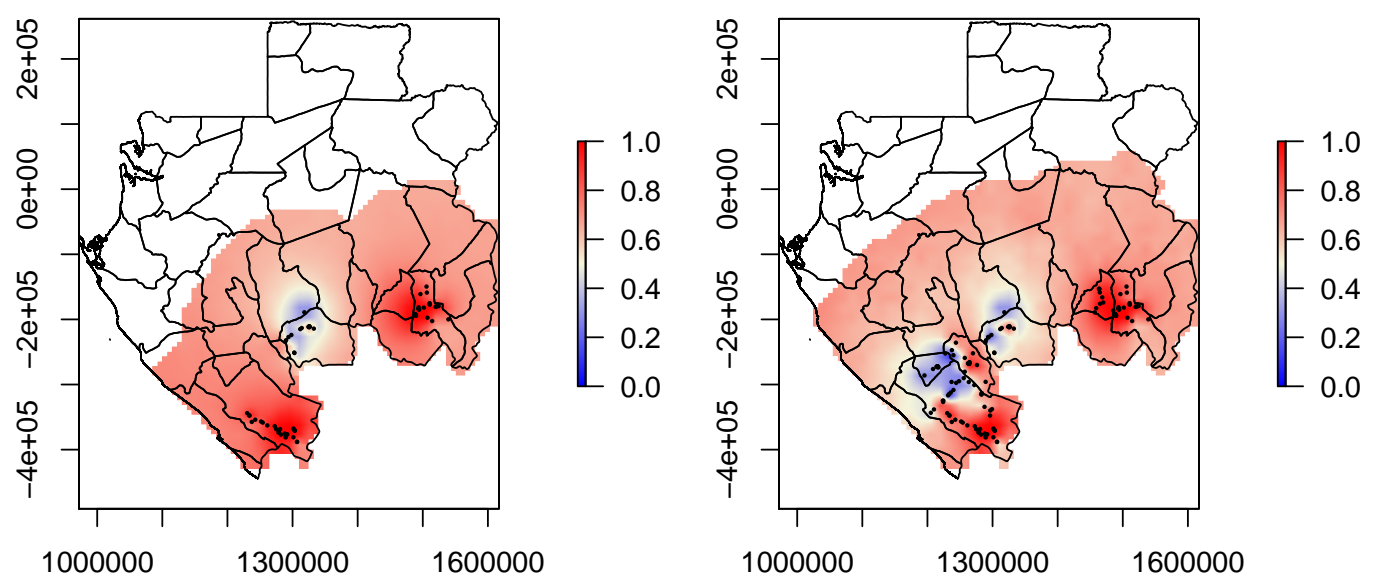

Figure 6: Maps of the predictive probability that at most $1 \%$ of individuals living at a particular location would give a Loa Scope estimate in excess of 20,000 microfilariae per ml. Solid dots show survey locations. The left-hand panel was constructed from the first round of surveys. In the right-hand panel the map has been updated to incorporate data from the second round of surveys.

$x$ by, in effect, allowing the pooling of information from neighbouring locations. As always, this extra information is not a free lunch; it is bought at the cost of making additional, albeit empirically test-able, modelling assumptions. Figure ?? shows a recent application of the Giorgi et al (2017) model to LoaScope data from Gabon. In this application, the agreed definition of "safe" was that with probability at least 0.95 , at most $1 \%$ of the individuals living at a location $x$ would give a LoaScope estimate in excess of 20,000 microfilariae per ml. After a first round of surveys concentrated in three distinct regions of Gabon, we constructed the predictive probability map of $T$ shown in the left-hand panel of Figure ??. This led to the decision to conduct a second round of surveys in the areas adjacent to the western and eastern surveyed regions, in the hope that this would extend the declared "safe" area. The result is shown in the right-hand panel of Figure ??, which does show a modest extension of the "safe" eastern area but also the emergence of a relatively unsafe area to the north of the western cluster of sampled locations. Note that in areas remote from any survey location predictive probabilities simply reflect the region-wide average properties of the data. For this reason, we caution against mapping such areas at all, so as to avoid the risk of over-interpretation. In Figure ?? we have chosen to restrict our predictions to locations for which the distance to the nearest data-location is less than the so-called practical range, i.e. the distance at which the fitted spatial correlation reaches the value 0.05 . 


\section{Combining information from multiple diagnostics}

As illustrated in Sections ?? and ??, the development of alternatives to blood-smear microscopy for diagnostic testing has been of crucial importance in the Africa-wide mapping of Loa loa risk. When different diagnostics have been used in the same geographical region, statistical models that can combine information from multiple diagnostics have the potential to make better use of all available data sources. Amoah et al (2018) introduce a geostatistical framework for combining data from multiple diagnostics and apply this to prevalence data obtained by blood-smear microscopy and by RAPLOA. They propose a bivariate geostatistical model that allows estimation of the calibration relationship between the two diagnostics, and uses this relationship to predict blood-smear microscopy-based prevalence in subregions where only the RAPLOA method has been used. Their proposed model can be expressed as

$$
\begin{aligned}
\operatorname{logit}\left\{P_{R L}(x)\right\} & =d^{\top}(x) \beta_{1}+S_{1}(x)+Z_{1} \\
\operatorname{logit}\left\{P_{M F}(x)\right\} & =d^{\top}(x) \beta_{2}+S_{2}(x)+Z_{2}+\alpha \operatorname{logit}\left\{P_{R L}(x)\right\}
\end{aligned}
$$

where $P_{R L}(x)$ and $P_{M F}(x)$ are, respectively, the underlying RAPLOA and blood-smear microsopy prevalences at location $x$. In this model, $S_{1}(x)$ and $S_{2}(x)$ are a pair of independent Gaussian processes that are used to account for unexplained spatial variation specific to each diagnostic. An advantage of this model over approaches that ignore the stochastic dependence between the two diagnostics is the increased precision of the resulting predictions. Against this, its practical utility in low-resource settings may be constrained by computational and data-storage requirements.

\section{Translating research into policy: decision-making un- der uncertainty}

An important implication of the shift from an onchocerciasis control programme to an elimination programme in Africa is that areas where ivermectin MDA was withheld, due to a low risk of blindness or skin disease (previously referred to as hypoendemic areas), now need to be reassessed and potentially treated if there is a potential for sustained transmission. The expansion of ivermectin MDA into areas where onchocerciasis sequelae are less prevalent brings into sharp focus the ethical dilemma of an acceptable SAE risk. How does one weigh the benefits of eliminating onchocerciasis in areas where the population has seldom felt the acute effects of the disease against the potential harm that treatment could inflict on some individuals? A comprehensive policy for the safe use of ivermectin has yet to be agreed. Any such policy would need to consider a complex mix of statistical, biomedical, social, economic and ethical issues. Test-and-not-treat undoubtedly gives the best protection against the occurrence of SAEs, which are not only catastrophic for the individuals concerned but harm the acceptability of ivermectin to their communities. Against this, with-holding treatment leaves untreated individuals at risk of onchocerciasis. Also, unless and until LoaScopes can be manufactured at scale, test-and-not-treat slows the Africa-wide roll-out of protective ivermectin treatment to regions where it is known that Loa loa is not co-endemic with onchocerciasis. One possible 
resolution is a hybrid policy in which spatial modelling can be used as a "first-pass" to determine if areas can be declared safe for MDA with ivermectin, based on a yet-to-be-determined acceptable level of SAE risk. Where the spatial modelling results indicate that the safety threshold has not been reached, individual village-by-village assessments using the Schlüter et al (2016) model, converging to the test-and-not-treat strategy with increasing SAE risk, would be required.

In the authors' opinion, statistical method can make two contributions to this complex issue. Firstly, given data and an agreed definition of "safe" at an agreed geographical scale, statistical modelling in conjunction with predictive infererence can deliver an honest assessment of the probability that the safety threshold has been reached. Secondly, when the available data are insufficent to resolve the safety issue, statistical design can inform where addtional data should be collected. Chipeta et al (2016) describe strategies for the adaptive design of sequential prevalence surveys to optimise a context-specific criterion.

\section{Conclusion}

At the time of writing, the "Loa loa problem" persists. Statistical modelling and inference can inform, but not resolve, the problem. Whatever policy decision is reached, it must be acknowledged that there is no such thing as zero risk. Balancing the remote risk of causing severe damage to a relatively small number of individuals against the likelihood of protecting much larger numbers against life-changing disease is not something that can be done using

only statistical or, more broadly, "scientific" arguments. Social context must also be taken into account and it is imperative that the affected countries be a leading voice in these deliberations. Nonethless, in the authors' opinion it is essential that any debate aimed at achieving consensus is informed by the use of statistical methods that make the best possible use of relevant data whilst acknowledging their necessary limitations.

\section{Acknowledgments}

This work received financial support from the UK Medical Research Council, and from the Coalition for Operational Research in Neglected Tropical Diseases (COR-NTD). COR-NTD is funded at The Task Force for Global Health primarily by the Bill \& Melinda Gates Foundation, by the United States Agency for International Development through its Neglected Tropical Diseases Program, and with UK aid from the British people. But the real heroes are the many field-workers who collect data under challenging conditions to provide the raw material for the statistical analyses summarised in the paper. 


\section{References}

Amoah, B., Giorgi, E., Diggle, P.J. (2018). A Geostatistical Framework for Combining Spatially Referenced Disease Prevalence Data from Multiple Diagnostics. Pre-print: arxiv.org/abs/1808.03141

Amazigo, U. (2008). The African Programme for Onchocerciasis Control (APOC). Annals of Tropical Medicine and Parasitology, 102 (Supplement 1), 19-22.

Boussinesq, M., Gardon, J., Gardon-Wendel, N., Kamgno, J., Ngoumou, P., and Chippaux, J.P. (1998). Three probable cases of Loa loa encephalopathy following Ivermectin treatment for Onchocerciasis. American Journal of Tropical Medicine and Hygiene, 58, 461-469.

Boussinesq, M., Gardon, J., Kamgno, J., Pion, S.D.S., Gardon-Wendel, N., and Chippaux, J.P. (2001). Relationships between the prevalence and intensity of Loa loa infection in the Central Province of Cameroon. Annals of Tropical Medicine and Parasitology, 95, 495-507.

Boussinesq, M., Gardon, J., Gardon-Wendel, N. and Chippaux, J. (2003). Clinical picture, epidemiology and outcome of Loa-associated serious adverse events related to mass ivermectin treatment of onchocerciasis in Cameroon. Filaria Journal, 2, 1-13.

Breslow, N.E. and Clayton, D.G. (1993). Approximate inference in generalized linear mixed models. Journal of the American Statistical Association 88, 9-25.

Centers for Disease Control and Prevention (1993). Recommendations of the International Task Force for Disease Eradication. MMWR Recommendations and Reports, 42, 1-38.

Chipeta, M., Terlouw, D.J., Phiri, K. and Diggle, P.J. (2016). Adaptive geostatistical design and analysis for sequential prevalence surveys. Spatial Statistics, 15, 70-84.

D’Ambrosio, M.V., Bakalar, M., Bennuru, S., Reber, C., Skandarajah, A., Nilsson, L., Switz, N., Kamgno, J., Pion S., Boussinesq, M., Nutman, T.B. and Fletcher, D.A. (2015). Point-ofcare quantification of blood-borne filarial parasites with a mobile phone microscope. Science Translational Medicine, 7, 286re4. doi: 10.1126/scitranslmed.aaa3480

Diggle, P.J., Moyeed, R.A. and Tawn, J.A. (1998). Model-based geostatistics (with Discussion). Applied Statistics 47 299-350.

Diggle, P.J., Thomson, M.C., Christensen, O.F., Rowlingson, B., Obsomer, V., Gardon, J., Wanji, S., Takougang, I., Enyong, P., Kamgno, J., Remme, H., Boussinesq, M. and Molyneux, D.H. (2007). Spatial modelling and prediction of Loa loa risk: decision making under uncertainty. Annals of Tropical Medicine and Parasitology, 101, 499-509.

Gardon, J., Gardon-Wendel, N., Denmarga-Ngangue, Kamgno, J., Chippaux, J.P. and Boussinesq, M. (1997). Serious reactions after mass treatment of onchocerciasis with ivermectin in an area endemic for Loa loa infection. Lancet, 350, 18-22

Giorgi, E., Schlüter, D.K. and Diggle, P.J. (2017). Bivariate geostatistical modelling of the relationship between Loa loa prevalence and intensity of infection. Environmetrics, 17, DOI: $10.1002 /$ env. 2447

Homeida, M., Braide, E., Elhassan, E., Amazigo, U. V., Liese, B., Benton, B., Noma, 
M., Etyaalé, D., Dadzie, K. Y., Kale, O. O. and Sékétéli, A. (2002). APOCs strategy of community-directed treatment with ivermectin (CDTI) and its potential for providing additional health services to the poorest populations. African Programme for Onchocerciasis Control. Annals of Tropical Medicine and Parasitology 96 (Supplement 1), S93-104.

Kamgno, J., Pion, S.D., Chesnais, C.B., Bakalar, M.H., DAmbrosio, M.V., Mackenzie, C.D., Nana-Djeunga, H.C., Gounoue-Kamkumo, R., Njitchouang, G-R, Nwane, P., TchatchuengMbouga, J.B., Wanji, S., Stolk, W.A., ; Fetcher, D.A., Klion, A.D., Nutman, T.B. and Boussinesq, M. (2017). A Test-and-not-treat strategy for Onchocerciasis in Loa loa-endemic areas. New England Journal of Medicine, 377, 2044-2052.

Schlüter, D.K., Ndeffo-Mbah, M.L., Takougang, I., Ukety, T., Wanji, S., Galvani, A.P. and Diggle, P.J. (2016). Using community-level prevalence of Loa loa infection to predict the proportion of highly-infected individuals: statistical modelling to support lymphatic filariasis elimination programs. PLoS Neglected Tropical Diseases, 10,12, e0005157. doi:10.1371/journal.pntd

Takougang, I., Meremikwu, M., Wanji, S., Yenshu, E.V., Aripko, B., Lamlenn, S.B., Eka, B.L., Enyong, P., Meli, J., Kale, O. and Remme, J.H. (2002). Rapid assessment method for prevalence and intensity of L. loa infection. Bulletin of the World Health Organisation, 80, $852-858$.

Thiele, E.A., Cama, V.A., Lakwo, T., Mekasha, S., Abanyie, F., Sleshi, M., Kebede, A. and Cantey, P.T. (2016). Detection of Onchocerca volvulus in skin snips by microscopy and real-time polymerase chain reaction: implications for monitoring and evaluation activities. American Journal of Tropical Medicine and Hygiene, 94, 906-911.

Thomson, M.C., Obsomer, V., Dunne, M., Connor, S.J. and Molyneux, D.H. (2000). Satellite mapping of Loa loa prevalence in relation to ivermectin use in west and central Africa. Lancet, 356, 1077-1078.

Thomson, M.C., Obsomer, V., Kamgno, J., Gardon, J., Wanji, S., Takougang, I., Enyong, P., Remme, J.H., Molyneux, D.H. and Boussinesq, M. (2004). Mapping the distribution of Loa loa in Cameroon in support of the African Programme for Onchocerciasis Control. Filaria Journal, 3, 7 .

Tobler, W. (1970). A computer movie simulating urban growth in the Detroit region. Economic Geography, 46, 234-240.

World Health Organization (2012). Accelerating work to overcome the global impact of neglected tropical diseases - a roadmap for implementation. Geneva: World Health Organization

World Health Organisation (2013). Progress towards eliminating onchocerciasis in the WHO region of the Americas: verification by WHO of elimination of transmission in Colombia. Weekly Epidemiologial Record, 88 (36), 381-385.

World Health Organisation (2014). Elimination of onchocerciasis in the WHO region of the Americas: Ecuadors progress towards verification of elimination. Weekly Epidemiologial Record, 89 (37), 401-405.

World Health Organisation (2015). Progress toward eliminating onchocerciasis in the WHO 
region of the Americas: verification of elimination of transmission in Mexico. Weekly Epidemiologial Record, 90 (43), 577-581.

World Health Organisation (2018). Weekly Epidemiological Report Weekly Epidemiological Record, 93, 633-648.

Zoure, H., Wanji, S., Noma, M., Amazigo, U., Diggle, P.J., Tekle, A. and Remme, J.H. (2011). The geographic distribution of Loa loa in Africa: results of large-scale implementation of the Rapid Assessment Procedure for Loiasis (RAPLOA). Public Library of Science: Neglected Tropical Diseases 5, (6): e1210.doi:10.1371/journal.pntd.0001210 